\title{
Can smartphone vibration provide a valid alternative to tuning forks following tympanomastoid surgery?
}

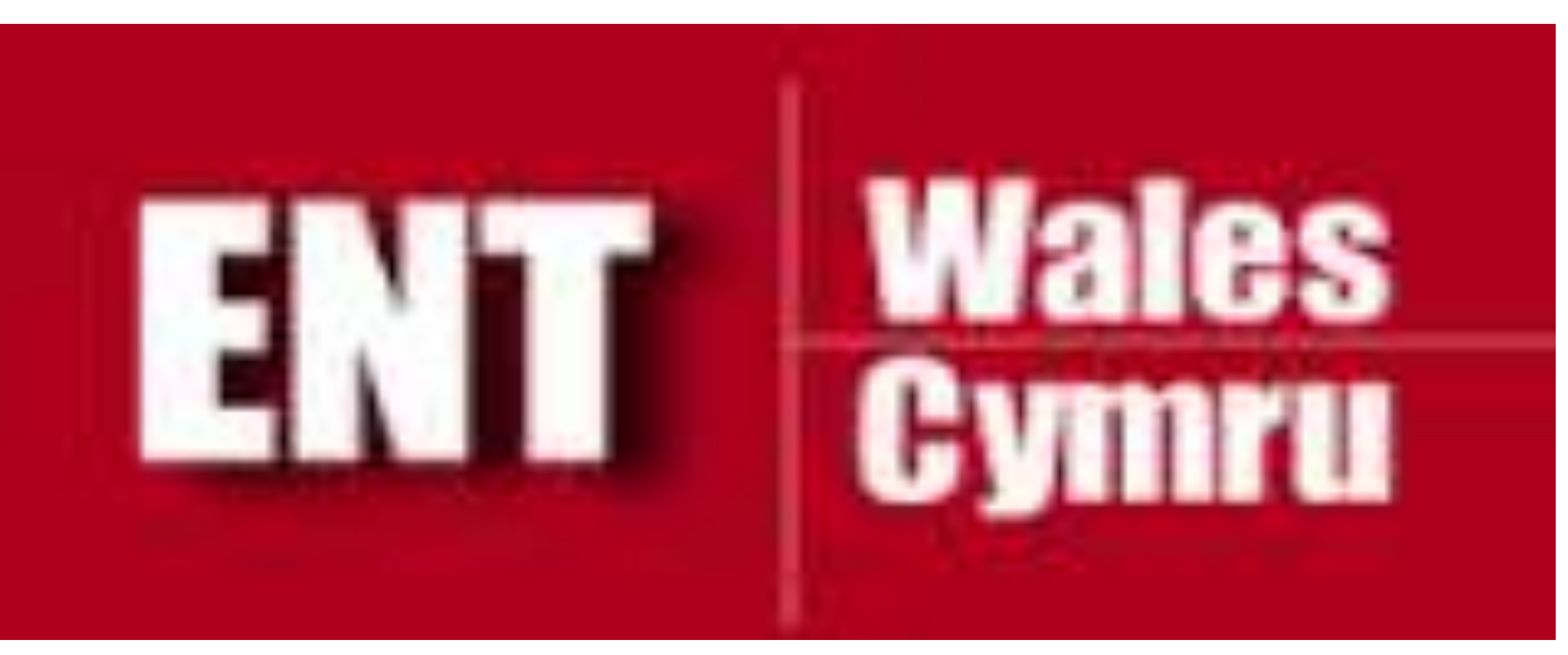

Mr. M Hopkins, Mr. D Owens
ENT Department
University Hospital of Wales
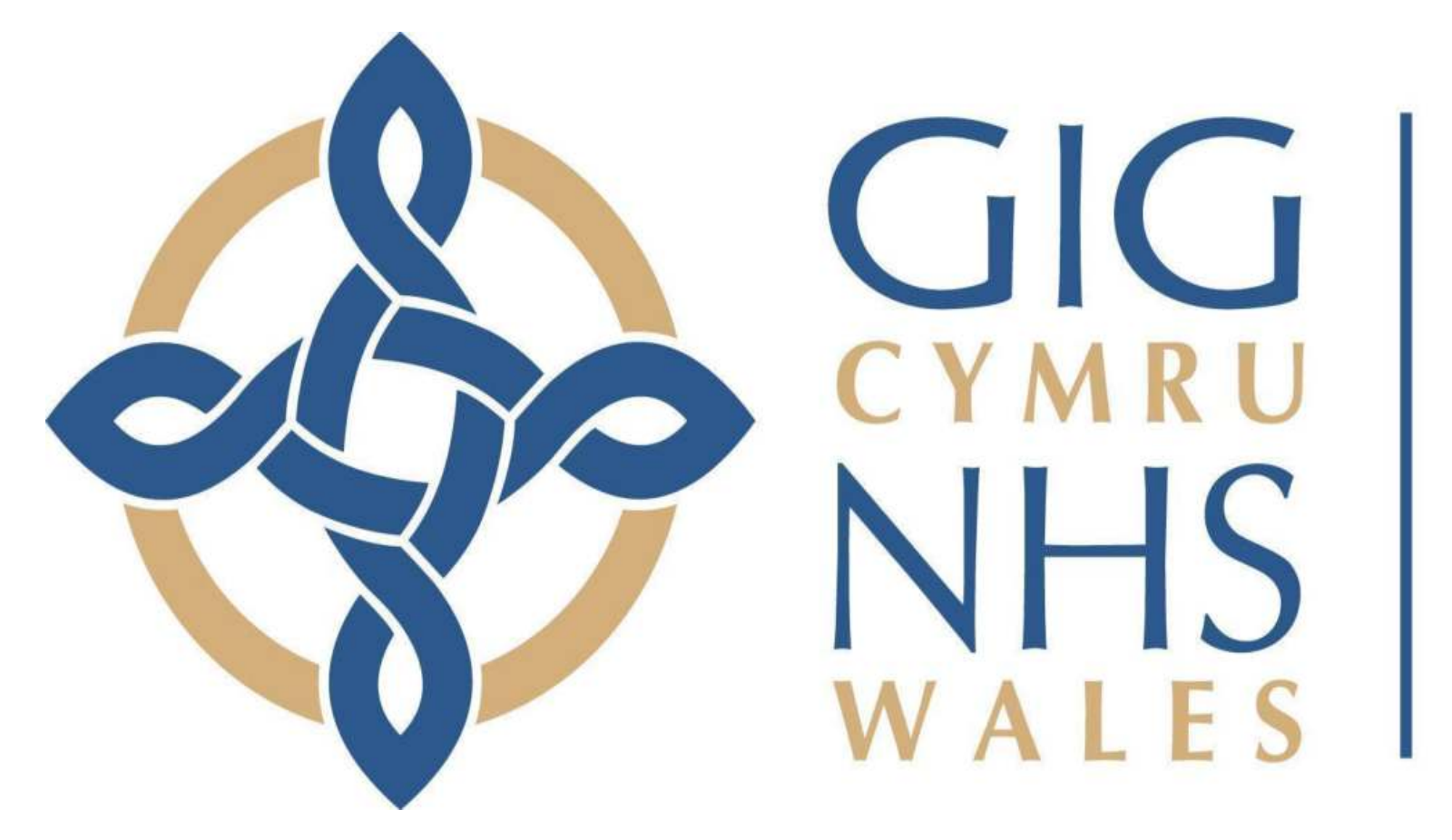

Bwrdd lechyd Prifysgol Caerdydd a'r Fro

Cardiff and Vale University Health Board

\section{Background and Aims}

The British Society of Otology stipulate that all patients undergoing tympanomastoid surgery should be assessed post-operatively for a 'dead ear' due to the risk of damage to the inner ear intra-

operatively ${ }^{1}$. This is typically assessed by performing Weber's test with a $512 \mathrm{~Hz}$ tuning fork to ensure sound is detected in the operated ear. Weber's test indicates a conductive hearing loss when the sound lateralises to the affected ear.. Regrettably, tuning forks are frequently inaccessible on hospital wards resulting in this examination frequently being omitted, and subsequently a patient missing a critical window of opportunity in which to resolve the problem.

Our objective was therefore to establish a suitable, more readily available alternative. We thus wanted to demonstrate that smartphone-based vibration applications provide equivalent accuracy to tuning forks when performing Weber's test for assessment inner ear function post-tympanomastoid surgery.
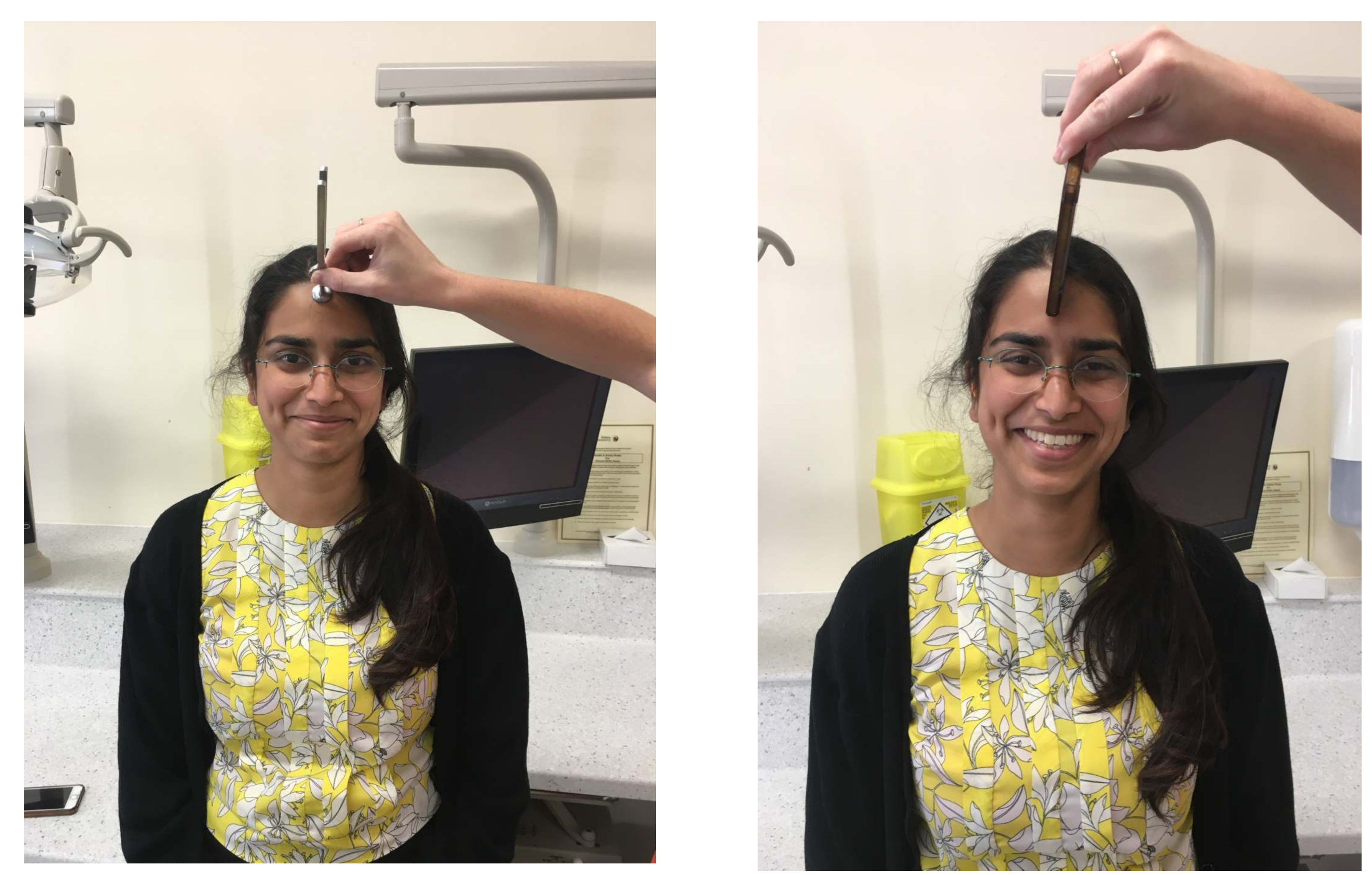

\section{Methods}

Data were collected prospectively on 20 lay participants with no underlying hearing impairment. Earplugs were used to simulate a conductive hearing loss, similar to that of packing post aural surgery. Both the right and left ears were tested with the iBrateMe $($ ) application on iPhone and a $512 \mathrm{~Hz}$ tuning fork, where the subject was asked to identify which ear the sound was perceived loudest. The edge of the phone was was cleaned with Chlorhexadine $2 \%$ wipes to ensure decontamination.

\section{Results}

On occlusion of the left ear canal, sound from the tuning fork lateralised to the left in 18/20 (90\%) cases. In $20 / 20(100 \%)$ cases sound lateralised to the left with the iPhone app (chi squared test, $p=0.82$ ). On Occlusion of the right ear, sound from the tuning fork laterilsed to the right in 19/20 (95\%) cases. In 19/20 (95\%) tests sound lateralised to the right with the iPhone (chi squared test, $p>0.999$ ). These findings substantiate the accuracy of Weber's test with tuning forks in determining a simple conductive hearing loss, furthermore they demonstrate the comparability of the vibration applications on the iPhone.

One limitation of the study is that we have used healthy subjects with otherwise normal hearing. In reality many patients have underlying conditions affecting their hearing which can have implications for the accuracy of tuning fork examinations ${ }^{2}$.
Weber's test performed when conductive hearing loss simulated in the left ear

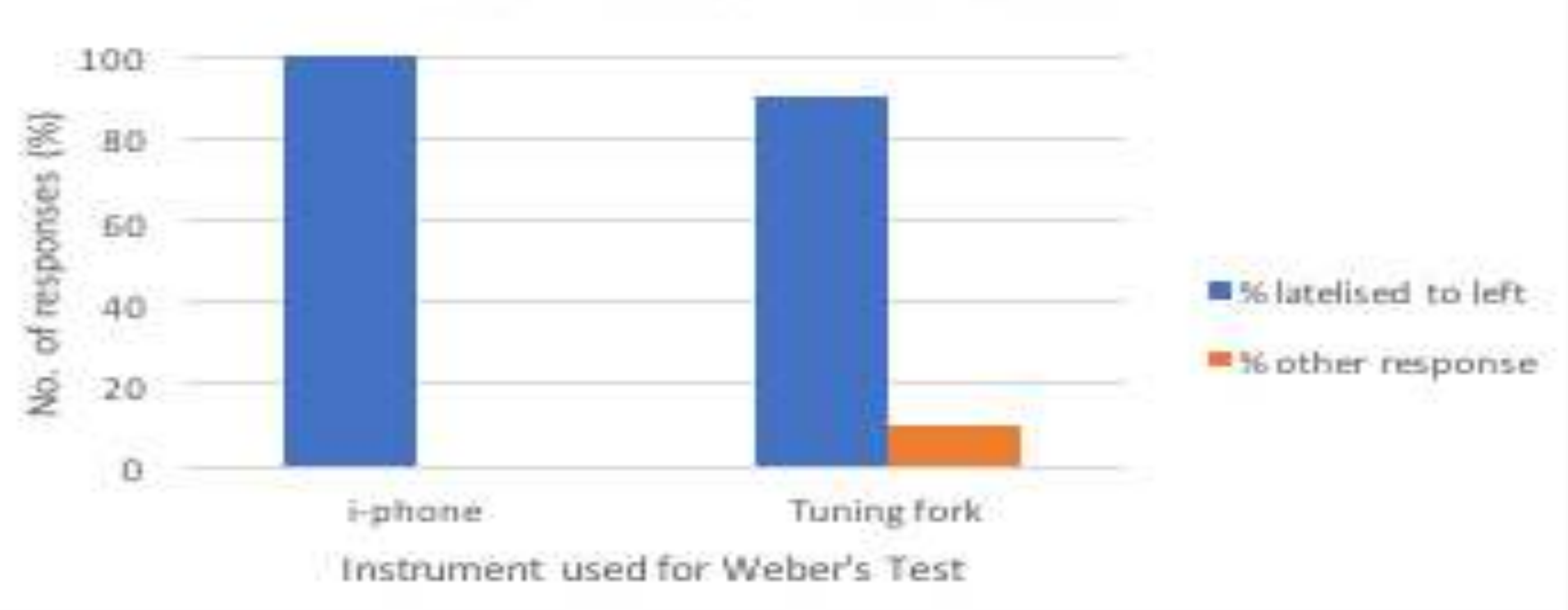

Weber's test performed when conductive hearing loss simulated in the right ear

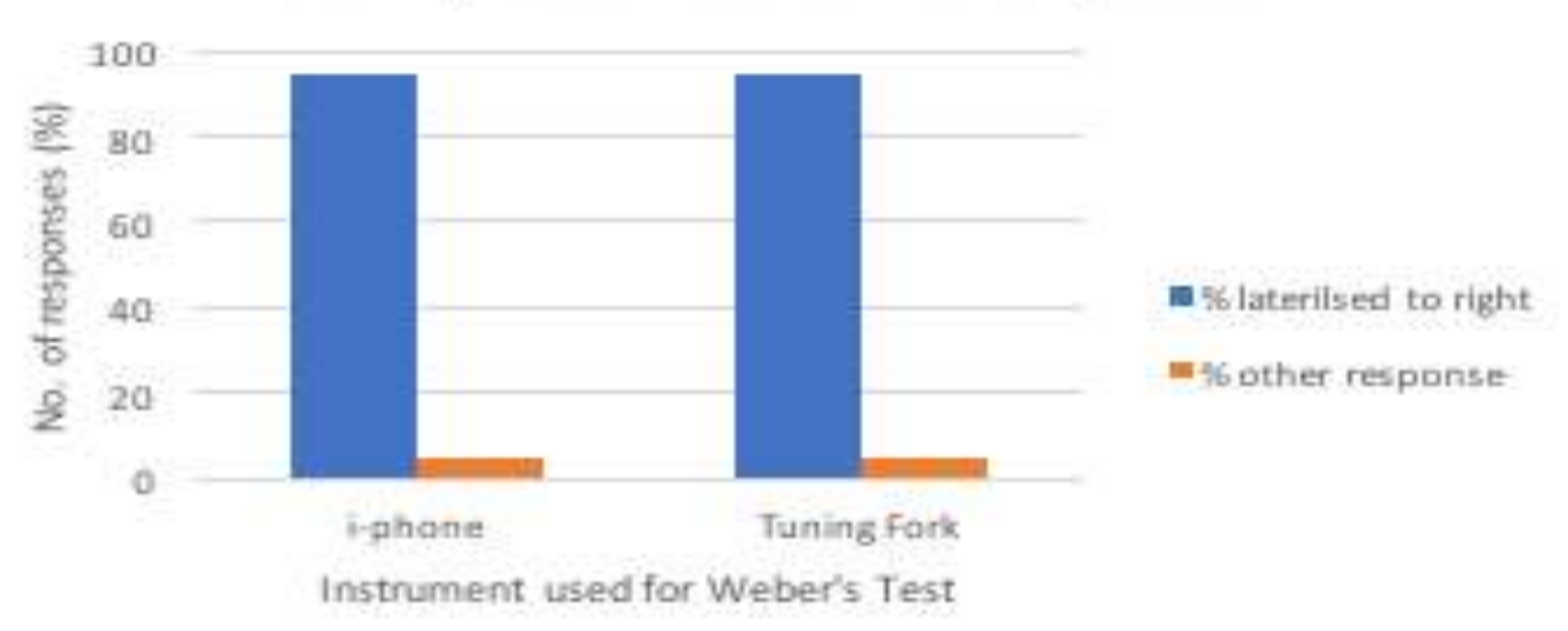

\section{Conclusions}

Smartphone-based vibration applications represent a viable, more accessible alternative to tuning forks when assessing inner ear function following tympanomastoid surgery.

\section{References}

1. lacovidou A et al. How reliable is the "scratch test" versus the weber test after tympanomastoid surgery. Otol Neurotol. 2014 Jun;35(5):762-3 